\title{
Numerical Investigation of the System-Matrix Method for Higher-Order Probe Correction in Spherical Near-Field Antenna Measurements
}

\author{
Thorkild B. Hansen \\ Seknion Inc., 2000 Commonwealth Ave, Boston, MA 01235, USA \\ Correspondence should be addressed to Thorkild B. Hansen, thorkild.hansen@att.net
}

Received 14 October 2011; Accepted 12 December 2011

Academic Editor: Claudio Gennarelli

Copyright (c) 2012 Thorkild B. Hansen. This is an open access article distributed under the Creative Commons Attribution License, which permits unrestricted use, distribution, and reproduction in any medium, provided the original work is properly cited.

\begin{abstract}
The system-matrix method for higher-order probe correction in spherical near-field scanning is based on a renormalized leastsquares approach in which the normal matrix closely resembles the identity matrix when most of the energy of the probe pattern resides in the first-order modes. This method will be "stressed-tested" in the present paper by employing probes for which up to $49 \%$ of the pattern energy resides in the higher-order modes. The condition number of the resulting normal matrix will be computed, and its "distance" from the identity matrix displayed. It is also shown how the condition number of the normal matrix can be further reduced.
\end{abstract}

\section{Introduction}

The standard theories for spherical near-field scanning of electromagnetic fields [1-5] hold for first-order probes that have $e^{ \pm i \phi}$ azimuthal pattern dependence only. For general higher-order two-port probes, which have unequal port patterns that contain modes $e^{i \mu \phi}$ with $\mu \neq \pm 1$, one cannot use these standard theories. (The term "higher-order mode," refers to all modes with $|\mu| \neq 1$, including the mode $\mu=$ 0 . Through $\chi$-scanning, the standard method [3] can deal with higher-order probes in special situations. However, this approach does not work in the situation of highest practical importance where the data consists of the output of a twoport higher-order probe.) Therefore, first-order probes have been preferred in spherical near-field scanning over the past 30 years.

Unfortunately, first-order probes are inherently narrowbanded, and thus a large number of different probes (each covering a narrow frequency band) are needed to measure the AUT over a broadband of frequencies. In practice, this means that wide-band characterizations become very time consuming because the spherical scan must be repeated many times. In addition, it takes a considerable amount of time to calibrate and change probes because precise alignment procedures are required.

Broadband probes that overcome all these problems do indeed exist, but their patterns contain higher-order modes. Typically, most of the probe-pattern energy is still in the first-order modes, but the higher-order modes are nevertheless strong enough to prevent a first-order correction scheme from being accurate. Therefore, the problem of developing probe-corrected theories for spherical near-field scanning that hold for higher-order probes has received considerable attention recently; see [6-8] and references therein.

In the present paper we shall evaluate the system-matrix method presented in [8], which uses a renormalized leastsquares approach to obtain a probe-corrected theory for general higher-order two-port probes in spherical scanning. The ports need not have identical patterns, and the theory holds for both a $\phi$-scan $(0 \leq \theta \leq \pi, 0 \leq \phi \leq 2 \pi)$ or a $\theta$-scan $(0 \leq \theta \leq 2 \pi, 0 \leq \phi \leq \pi)$, and the scan points are obtained by rectangular $\theta-\phi$ sampling. The spherical expansion coefficients of the antenna under test (AUT) are determined by solving a linear system of equations involving a square normal matrix. This solution can be achieved in 
two alternative ways: (i) an iterative method that employs the conjugate gradient method and (ii) a direct method where the normal matrix is inverted explicitly. Both versions require $O\left(N^{3}\right)$ operations with very little overhead. Here, $N$ is the truncation number in the spherical expansion of the AUT field; see Section 2. Through a validation in [8] with experimental data, it was demonstrated that 29280 spherical expansion coefficients of an AUT can be computed in 8 seconds on a PC running Matlab.

We will "stress-test" the method by using probes for which up to $49 \%$ of the pattern energy resides in the higher-order modes. We compute the condition number and other statistics of the resulting normal matrix. Since the AUT spherical expansion coefficients can be obtained more rapidly when the normal matrix is close to the identity matrix, we shall investigate ways to "move" the normal matrix closer to the identity matrix.

The paper is organized as follows. In Section 2 we show how the AUT far-field pattern can be expressed in terms of the transverse spherical vector-wave functions and spherical expansion coefficients. Section 3 describes the renormalized least-squares solution from [8] that will be examined in this paper. In Section 4 we introduce the higher-order test probe that will be used in the numerical examples. Section 5 presents numerical results that include the evaluation of the condition number of the normal matrix for various probes and matrix truncations. In addition, we compute spherical expansion coefficients of a simulated AUT using different truncations of the normal matrix to demonstrate that the higher-order method is exact to machine precision. Moreover, we show that the method is very stable in the presence of noise, as would be expected since the normal matrix is extremely well conditioned. Section 6 presents conclusions. Throughout, we assume time-harmonic fields that have $e^{-i \omega t}$ time dependence with $\omega>0$.

\section{Vector-Wave Expansion of the Electric Field of the AUT}

In this section we express the AUT far-field pattern in terms of spherical expansion coefficients and transverse spherical vector-wave functions. The standard spherical coordinates $(r, \theta, \phi)$ with unit vectors given by

$$
\begin{aligned}
& \hat{\mathbf{r}}=\hat{\mathbf{x}} \cos \phi \sin \theta+\hat{\mathbf{y}} \sin \phi \sin \theta+\hat{\mathbf{z}} \cos \theta \\
& \hat{\boldsymbol{\theta}}=\hat{\mathbf{x}} \cos \theta \cos \phi+\hat{\mathbf{y}} \cos \theta \sin \phi-\hat{\mathbf{z}} \sin \theta \\
& \hat{\boldsymbol{\phi}}=-\hat{\mathbf{x}} \sin \phi+\hat{\mathbf{y}} \cos \phi
\end{aligned}
$$

will be used throughout. Here, the unit vectors for the rectangular coordinates $(x, y, z)$ are $\hat{\mathbf{x}}, \hat{\mathbf{y}}$, and $\hat{\mathbf{z}}$. The AUT is shown in Figure 1 inside the scan sphere with radius $R$. Moreover, the AUT minimum-sphere radius $R_{\min }$ is defined such that the maximum (supremum) value of the coordinate $r$ for all points on the AUT equals $R_{\min }$.

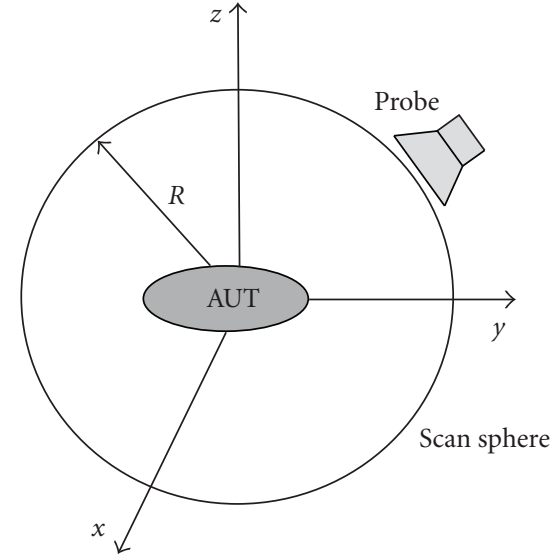

FIgURE 1: A probe with its reference point on the scan sphere of radius $R$ and its normal pointing towards the origin measures the field of the AUT.

The electric and magnetic AUT far fields can be expressed in terms of the far-field pattern $\mathscr{F}(\theta, \phi)$ as [9, page 110]

$$
\begin{aligned}
\mathbf{E}(\mathbf{r}) & \sim \mathcal{F}(\theta, \phi) \frac{e^{i k r}}{r}, \\
\mathbf{H}(\mathbf{r}) & \sim \sqrt{\frac{\epsilon_{0}}{\mu_{0}}} \hat{\mathbf{r}} \times \mathscr{F}(\theta, \phi) \frac{e^{i k r}}{r},
\end{aligned}
$$

where $\mu_{0}$ and $\epsilon_{0}$ are the free-space permeability and permittivity, respectively. Also, $k=\omega \sqrt{\mu_{0} \epsilon_{0}}$ is the wavenumber. The far-field pattern determined from the electric field as

$$
\mathscr{F}(\theta, \phi)=\lim _{r \rightarrow \infty} r e^{-i k r} \mathbf{E}(r \hat{\mathbf{r}})
$$

can be expressed in terms of the transverse vector-wave functions $\mathbf{M}_{n m}$ and $\mathbf{N}_{n m}$ as

$$
\begin{aligned}
\mathscr{F}(\theta, \phi)= & \frac{1}{k} \sum_{n=1}^{N} \sum_{m=-n}^{n} i^{-n-1} \\
& \times\left[A_{n m} \mathbf{M}_{n m}(\theta, \phi)+i B_{n m} \mathbf{N}_{n m}(\theta, \phi)\right],
\end{aligned}
$$

where $A_{n m}$ and $B_{n m}$ are the spherical expansion coefficients of the AUT, satisfying $A_{n m}=0$ and $B_{n m}=0$ when $|m|>n$. The truncation number $N$ is determined from the radius of the minimum sphere as

$$
N=k R_{\min }+\gamma\left(k R_{\min }\right)^{1 / 3}
$$

where the constant $\gamma$ determines the number of digits of accuracy achieved [10, Section 3.4.2]. The transverse vectorwave functions can be expressed in terms of the spherical harmonic $Y_{n m}(\theta, \phi)[11$, page 99] as [11, pages 742-746]

$$
\mathbf{M}_{n m}(\theta, \phi)=\hat{\boldsymbol{\theta}} \frac{i m Y_{n m}(\theta, \phi)}{\sqrt{n(n+1)} \sin \theta}-\hat{\boldsymbol{\phi}} \frac{(\partial / \partial \theta) Y_{n m}(\theta, \phi)}{\sqrt{n(n+1)}}
$$

and $\mathbf{N}_{n m}(\theta, \phi)=\hat{\mathbf{r}} \times \mathbf{M}_{n m}(\theta, \phi)$. The formula (4) makes it possible to compute the AUT far-field pattern in any direction from the spherical expansion coefficients $A_{n m}$ and $B_{n m}$. The goal of this paper is to evaluate the method presented in [8] for computing $A_{n m}$ and $B_{n m}$ from the output of a higher-order probe on the scan sphere. 


\section{Renormalized Least-Squares Formulation for Higher-Order Probes}

In this section we briefly outline the method derived in detail in [8]. By applying Fourier series expansions for the $d$ functions in Jensen's transmission formulas [1-3], one finds that the output of an arbitrary probe on the scan sphere can be expressed as the Fourier series

$$
\begin{aligned}
V^{(j)}(\theta, \phi)= & \sum_{q=-N}^{N} \sum_{m=-N}^{N}\left[\sum_{n=1}^{N} A_{n m} \mathcal{A}_{m q n}^{(j)}+\sum_{n=1}^{N} B_{n m} \mathcal{B}_{m q n}^{(j)}\right] \\
& \times e^{i m \phi} e^{i q \theta}+\epsilon(\theta, \phi),
\end{aligned}
$$

where $\boldsymbol{\epsilon}(\theta, \phi)$ accounts for positioning errors, reflections from chamber walls, inaccuracies in the probe model, and so forth. Here, the index $j$, which can take the values 1 or 2, represents the two ports of the higher-order probe. Equation (7) holds only when multiple interactions between the probe and AUT are negligible and defines a function that is $2 \pi$-periodic with respect to both $\theta$ and $\phi$.

The probe Fourier coefficient $\mathcal{A}_{\text {mqn }}^{(j)}$ is the Fourier coefficient for the output of Port no. $j$ when $A_{n^{\prime} m^{\prime}}=\delta_{n n^{\prime}} \delta_{m m^{\prime}}$ and $B_{n^{\prime} m^{\prime}}=0$. Similarly, the probe Fourier coefficient $\mathscr{B}_{m q n}^{(j)}$ is the Fourier coefficient for the output of Port no. $j$ when $B_{n^{\prime} m^{\prime}}=\delta_{n n^{\prime}} \delta_{m m^{\prime}}$ and $A_{n^{\prime} m^{\prime}}=0$. We note that $\mathscr{A}_{m q n}^{(j)}=0$ and $\mathcal{B}_{m q n}^{(j)}=0$ when either $|q|>n$ or $|m|>n$. Here, $\delta_{m m^{\prime}}=1$ if $m=m^{\prime}$ and zero otherwise.

It was shown in [8] how the probe Fourier coefficients $\mathcal{A}_{m q n}^{(j)}$ and $\mathscr{B}_{m q n}^{(j)}$ can be computed from the far-field pattern of an arbitrary probe. In the present paper we employ a higher-order probe made from Hertzian dipoles (see Section 4), so the probe output is determined directly from the field on the scan sphere. Hence, we do not need to go through the full formulation developed in [8]. To compute $\mathcal{A}_{m q n}^{(j)}$ and $\mathcal{B}_{m q n}^{(j)}$, we let $V_{A_{n m}}^{(j)}(\theta, \phi)$ denote the higher-order probe output due to a source with $A_{n^{\prime} m^{\prime}}=$ $\delta_{n n^{\prime}} \delta_{m m^{\prime}}$ and $B_{n^{\prime} m^{\prime}}=0$. Similarly, $V_{B_{n m}}^{(j)}(\theta, \phi)$ is the higherorder probe output due to a source with $A_{n^{\prime} m^{\prime}}=0$ and $B_{n^{\prime} m^{\prime}}=\delta_{n n^{\prime}} \delta_{m m^{\prime}}$. The Fourier expansion (7) implies that $V_{A_{n m}}^{(j)}(\theta, \phi)$ and $V_{B_{n m}}^{(j)}(\theta, \phi)$ can be computed at $\phi=0$ through

$$
\begin{aligned}
& V_{A_{n m}}^{(j)}(\theta, 0)=\sum_{q=-n}^{n} \mathcal{A}_{m q n}^{(j)} e^{i q \theta}, \\
& V_{B_{n m}}^{(j)}(\theta, 0)=\sum_{q=-n}^{n} \mathcal{B}_{m q n}^{(j)} e^{i q \theta} .
\end{aligned}
$$

We extend the $\theta$ variable to the entire range $0 \leq$ $\theta \leq 2 \pi$ by noting that the spherical unit vector satisfies $\widehat{\mathbf{r}}(\theta, \phi)=\hat{\mathbf{r}}(2 \pi-\theta, \phi+\pi)$. The standard sampling theorem for bandlimited periodic functions then gives the

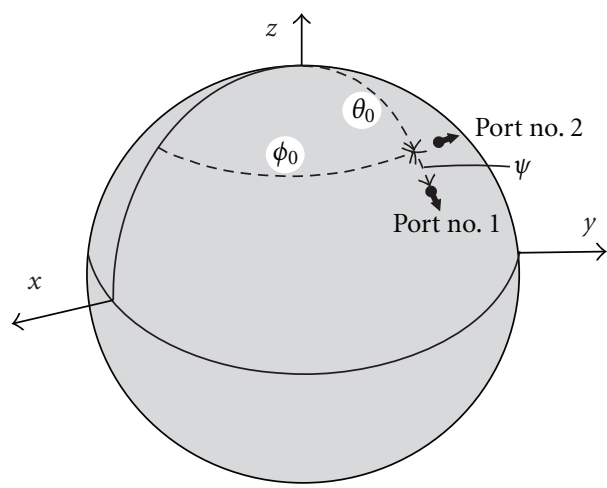

FIgURE 2: The higher-order test probe with its reference point at $\left(\theta_{0}, \phi_{0}\right)$ on the scan sphere of radius $R$. The output of Port no. 1 is $V^{(1)}\left(\theta_{0}, \phi_{0}\right)=E_{\theta}\left(R, \theta_{0}+\psi, \phi_{0}\right)$, and the output of Port no. 2 is $V^{(2)}\left(\theta_{0}, \phi_{0}\right)$ given by (19).

following exact expressions for the probe Fourier coefficients

$$
\begin{aligned}
& \mathcal{A}_{m q n}^{(j)}=\frac{1}{2 n+2} \sum_{s=0}^{2 n+1} V_{A_{n m}}^{(j)}\left(\frac{\pi s}{(n+1)}, 0\right) e^{-i \pi q s /(n+1)}, \\
& \mathcal{B}_{m q n}^{(j)}=\frac{1}{2 n+2} \sum_{s=0}^{2 n+1} V_{B_{n m}}^{(j)}\left(\frac{\pi s}{(n+1)}, 0\right) e^{-i \pi q s /(n+1)},
\end{aligned}
$$

where $q=-n, \ldots, 0, \ldots, n$.

Having completed the description of the Fourier formula (7), we next convert it to matrix form. Let the unknown AUT expansion coefficients $A_{n m}$ and $B_{n m}$ be given by the column vector $\mathbf{T}$ with length $O\left(2 N^{2}\right)$. Hence, the two-dimensional arrays $A_{n m}$ and $B_{n m}$ are collapsed into one-dimensional arrays and combined in a column vector. Similarly, we let the sampled higher-order probe output $V^{(j)}((t-1) \Delta \theta,(p-$ 1) $\Delta \phi$ ) be given by the column vector $\mathbf{W}$ of length $O\left(4 N^{2}\right)$, where $t$ and $p$ are integers. The sphere is discretized through $\theta_{t}=(t-1) \Delta \theta$ and $\phi_{p}=(p-1) \Delta \phi$, where $\Delta \theta$ and $\Delta \phi$ are chosen as usual to be less than or equal to $2 \pi /(2 N+1)$.

The formula (7) defines the probe matrix $\overline{\mathcal{P}}$, so that the probe output is

$$
\mathrm{W}=\overline{\mathscr{P}} \mathrm{T}
$$

which is the nonsquare system-matrix equation with more equations than unknowns for determining $\mathbf{T}$ for a given $\mathbf{W}$. The probe matrix $\overline{\mathcal{P}}$ has the form

$$
\overline{\mathscr{P}}=\overline{\mathbf{F}} \overline{\mathbf{Q}}
$$

where matrix multiplications with $\overline{\mathbf{F}}$ can be performed using a 2D FFT algorithm and $\overline{\mathbf{Q}}$ performs the summation over $n$ in the square brackets of (7).

We developed in [8] the renormalized least-squares solution to (10) given by

$$
\left(\overline{\mathbf{p}}^{-1}\right)^{H} \overline{\mathbf{Q}}^{H} \overline{\mathbf{F}}^{H} \overline{\boldsymbol{\tau}} \mathbf{W}=\overline{\mathbf{M}}[\overline{\mathbf{p}} \mathbf{T}],
$$

where the square matrix $\overline{\mathbf{M}}$ is

$$
\overline{\mathbf{M}}=\left(\overline{\mathbf{p}}^{-1}\right)^{H} \overline{\mathbf{Q}}^{H} \overline{\mathbf{F}}^{H} \overline{\boldsymbol{\tau}} \overline{\mathbf{F}} \overline{\mathbf{Q}} \overline{\mathbf{p}}^{-1} .
$$




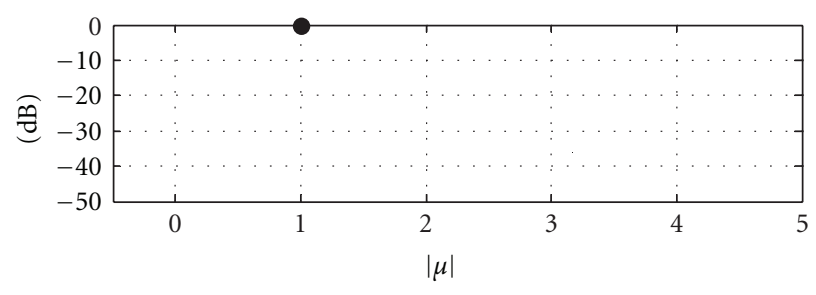

- $\psi=0 \mathrm{deg}, 100 \%$ of power in $|\mu|=1$ modes

(a)

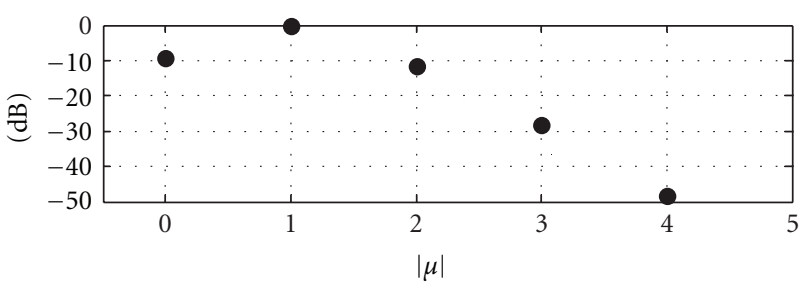

- $\psi=1 \mathrm{deg}, 84 \%$ of power in $|\mu|=1$ modes

(c)

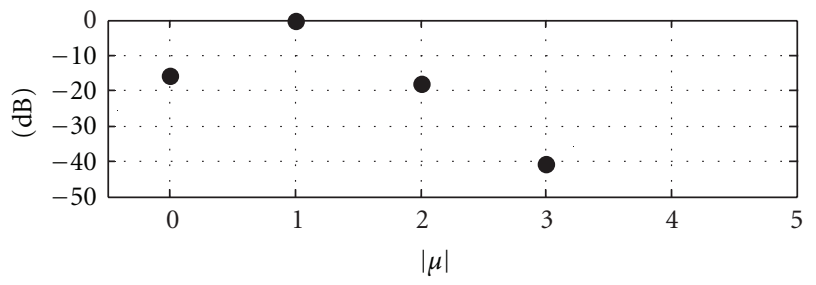

- $\psi=0.5 \mathrm{deg}, 96 \%$ of power in $|\mu|=1$ modes

(b)

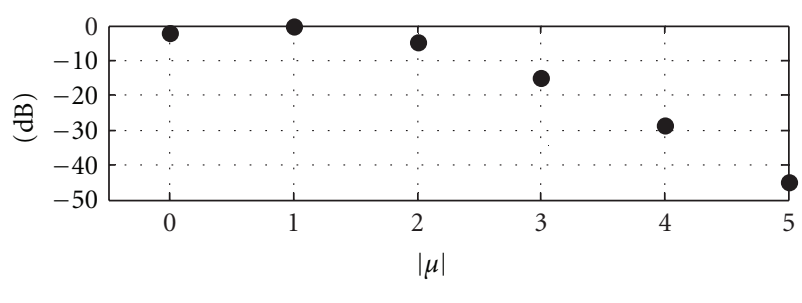

- $\psi=2 \mathrm{deg}, 51 \%$ of power in $|\mu|=1$ modes

(d)

Figure 3: The power in the modes of test probes with $\psi$ equal to $0^{\circ}, 0.5^{\circ}, 1^{\circ}$, and $2^{\circ}$. The power for each value of $|\mu|=K$ is obtained by summing the power of all modes with $\mu= \pm K$. The plots are normalized so that the power in the $\mu= \pm 1$ modes is $0 \mathrm{~dB}$.

Here $\overline{\boldsymbol{\tau}}$ is a diagonal matrix containing the surface element $\Delta \phi \Delta \theta|\sin [(p-1) \Delta \theta]|$ and $\overline{\mathbf{p}}$ is the first-order correction factor, which is a diagonal matrix that represents a first-order correction. The renormalization achieved with $\overline{\mathbf{p}}$ results in $\overline{\mathbf{M}}$ being almost equal to the identity matrix when most of the probe-pattern energy is in the first-order modes.

In this paper we use the Hertzian-dipole first-order correction factor with elements $U h_{n}^{(1)}(k R)$ and $U g_{n}^{(1)}(k R)$, where $\mathcal{U}$ is the constant determined from the probe Fourier coefficients as

$$
\mathcal{U}=-\sqrt{\frac{8 \pi}{3}} \frac{1}{g_{1}(k R)} \sum_{q=-1}^{1} \mathcal{B}_{0 q 1}^{(1)} e^{i q \pi / 2},
$$

where $h_{n}^{(1)}(z)$ is the spherical Hankel function [11, page 740] and

$$
g_{n}^{(1)}(z)=\frac{1}{z} \frac{\partial}{\partial z}\left[z h_{n}^{(1)}(z)\right] .
$$

As discussed in [8], one can compute an optimal first-order correction factor, which may result in more well-conditioned normal matrices than the Hertzian-dipole correction factor used in this paper.

The choice of first-order correction factor $\overline{\mathbf{p}}$ does not change the outcome of the computation of the AUT expansion coefficients. It merely affects the matrix $\overline{\mathbf{M}}$ and of course the left side of (12). A good correction factor results in $\overline{\mathbf{M}}$ being close to the identity matrix. Equation (12) can be solved with respect to $\overline{\mathbf{p}} \mathbf{T}$ using a linear iterative solver such as the conjugate gradient method, regardless of how closely $\overline{\mathbf{M}}$ resembles the identity matrix. However, when $\overline{\mathbf{M}} \approx \overline{\mathbf{I}}$, we can obtain a closed-form solution by using the Neumann series. The computation scheme presented here has computational complexity $N^{3}[8]$, and the matrix $\overline{\mathbf{M}}$ is never computed explicitly.

\section{Higher-Order Test Probe}

We shall use an offset Hertzian dipole as the test probe for evaluating the computation scheme. The probe has two ports that each equal the tangential components of the electric field on the scan sphere as shown in Figure 2.

The higher-order test probe is specified by the offset angle $\psi$. Specifically, when the reference point of the probe is at $\left(\theta_{0}, \phi_{0}\right)$, the output of Port no. 1 is the $\theta$ component of the electric field at $\mathbf{r}_{1}\left(\theta_{0}, \phi_{0}\right)=R \widehat{\mathbf{r}}_{1}=R \widehat{\mathbf{r}}\left(\theta_{0}+\psi, \phi_{0}\right)$ :

$$
\begin{aligned}
V^{(1)}\left(\theta_{0}, \phi_{0}\right) & =\hat{\boldsymbol{\theta}}\left(\theta_{0}+\psi, \phi_{0}\right) \cdot \mathbf{E}\left(\mathbf{r}_{1}\left(\theta_{0}, \phi_{0}\right)\right) \\
& =E_{\theta}\left(R, \theta_{0}+\psi, \phi_{0}\right) .
\end{aligned}
$$

The output of Port no. 2 is obtained by rotating the Hertzian dipole of Port no. 190 degrees with respect to the unit vector $\widehat{\mathbf{r}}\left(\theta_{0}, \phi_{0}\right)$. To determine the location of the dipole for Port no. 2, we introduce two lengths $\Delta$ and $L$ so that $(R+\Delta) \widehat{\mathbf{r}}_{1}=R \widehat{\mathbf{r}}_{0}+L \widehat{\boldsymbol{\theta}}_{0}$, where $\widehat{\mathbf{r}}_{0}$ and $\widehat{\boldsymbol{\theta}}_{0}$ are the unit vectors corresponding to $\left(\theta_{0}, \phi_{0}\right)$. Since $\hat{\mathbf{r}}_{1} \cdot \hat{\mathbf{r}}_{0}=\cos \psi$, we have $(R+\Delta) \cos \psi=R$ and $(R+\Delta)^{2}=R^{2}+L^{2}$, so $\mathrm{L}=R \tan \psi$. We can now determine the location $\mathbf{r}_{2}$ of the dipole for Port no. 2 by noting that $(R+\Delta) \widehat{\mathbf{r}}_{2}=R \widehat{\mathbf{r}}_{0}+L \hat{\boldsymbol{\phi}}_{0}$ which gives

$$
\mathbf{r}_{2}\left(\theta_{0}, \phi_{0}\right)=R \hat{\mathbf{r}}_{2}=R\left(\hat{\mathbf{r}}_{0} \cos \psi+\hat{\phi}_{0} \sin \psi\right)
$$



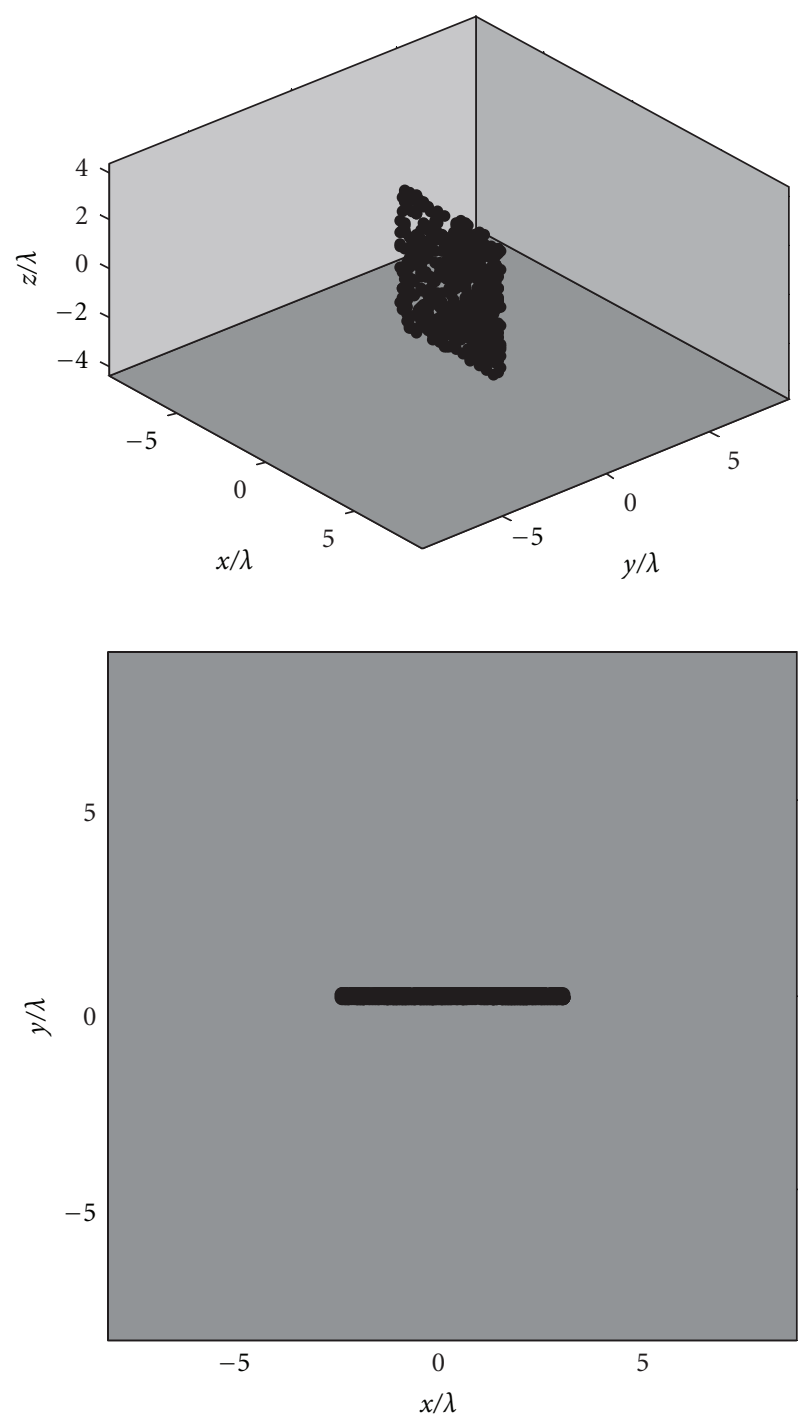

FIgURE 4: The AUT consisting of $500 z$-directed Hertzian dipoles randomly distributed in a box with dimensions $-2.8 \lambda<x<2.8 \lambda$, $-0.03 \lambda<y<0.03 \lambda$, and $-2.8 \lambda<z<2.8 \lambda$, so that $R_{\min }=4 \lambda$.

where $\hat{\boldsymbol{\phi}}_{0}=\hat{\boldsymbol{\phi}}\left(\theta_{0}, \phi_{0}\right)$. The direction $\hat{\boldsymbol{\alpha}}_{2}$ of the dipole for Port no. 2 is tangential to the sphere at $\mathbf{r}_{2}$ and lies in the plane containing the origin, $\hat{\mathbf{r}}_{2}$, and $\widehat{\mathbf{r}}_{0}$ :

$$
\widehat{\boldsymbol{\alpha}}_{2}\left(\theta_{0}, \phi_{0}\right)=\frac{1}{\sin \psi}\left(\widehat{\mathbf{r}}_{2} \cos \psi-\widehat{\mathbf{r}}_{0}\right)=\widehat{\phi}_{0} \cos \psi-\widehat{\mathbf{r}}_{0} \sin \psi
$$

so that the output of Port no. 2 is

$$
V^{(2)}\left(\theta_{0}, \phi_{0}\right)=\widehat{\boldsymbol{\alpha}}_{2}\left(\theta_{0}, \phi_{0}\right) \cdot \mathbf{E}\left(\mathbf{r}_{2}\left(\theta_{0}, \phi_{0}\right)\right) .
$$

When $\psi=0$, the test probe reduces to a first-order probe with outputs equal to the tangential components of the electric field at $\left(\theta_{0}, \phi_{0}\right)$. From [8, equation (1)] it is seen that

$$
\begin{aligned}
& V_{A_{n m}}^{(1)}(\theta, 0)=\hat{\boldsymbol{\theta}}(\theta+\psi, 0) \cdot \mathbf{M}_{n m}(\theta+\psi, 0) h_{n}^{(1)}(k R), \\
& V_{\mathrm{B}_{n m}}^{(1)}(\theta, 0)=\hat{\boldsymbol{\theta}}(\theta+\psi, 0) \cdot \mathbf{N}_{n m}(\theta+\psi, 0) g_{n}(k R),
\end{aligned}
$$

$$
\begin{aligned}
& V_{A_{n m}}^{(2)}(\theta, 0)=\widehat{\boldsymbol{\alpha}}_{2}(\theta, 0) \cdot \mathbf{M}_{n m}\left(\widehat{\mathbf{r}}_{2}(\theta, 0)\right) h_{n}^{(1)}(k R), \\
& V_{B_{n m}}^{(2)}(\theta, 0)=\widehat{\boldsymbol{\alpha}}_{2}(\theta, 0) \cdot \mathbf{N}_{n m}\left(\widehat{\mathbf{r}}_{2}(\theta, 0)\right) g_{n}(k R)
\end{aligned}
$$

so that the probe Fourier coefficients $\mathcal{A}_{m q n}^{(j)}$ and $\mathscr{B}_{m q n}^{(j)}$ can be determined from (9).

We shall perform the evaluation of the higher-order computation method using a scan sphere of radius $6 \lambda$ with a minimum radius of the AUT of $R_{\min }=4 \lambda(\lambda$ is the wavelength). We employ test probes with $\psi$ equal to $0^{\circ}$, $0.5^{\circ}, 1^{\circ}$, and $2^{\circ}$. Figure 3 shows the power spectrum of these probes. The value at $|\mu|=K$ is obtained by summing the power of all modes with $\mu= \pm K$. The plots are normalized so that the power in the $\mu= \pm 1$ modes is $0 \mathrm{~dB}$.

Table 1 shows the power content of first-order modes as a function of $\psi$. The commercially available higher-order probe that was used in the experiential validation in [8] had $97 \%$ of its power in the first-order modes. Nevertheless, the remaining $3 \%$ of the power residing in the higherorder modes made first-order probe correction completely inadequate. For one of the field components, the AUT farfield pattern error was nearly $10 \mathrm{~dB}$ near the main direction; see [8, Figure 5], hence, the need for higher-order methods even when almost all the energy is in the first-order modes.

\section{Evaluation of the Normal Matrix}

With an $R=6 \lambda$ scan sphere and an AUT minimum radius of $R_{\min }=4 \lambda$, we compute in this section the normal matrix $\overline{\mathbf{M}}$ corresponding to each of the four probes with $\psi$ equal to $0^{\circ}$, $0.5^{\circ}, 1^{\circ}$, and $2^{\circ}$ shown in Figure 3. With $\gamma=7$, the truncation formula (5) gives $N=46$, and we sample the sphere with 48 equally spaced samples in $\theta$ and 96 equally spaced samples in $\phi$. In actual processing of near-field scanning data, one should never compute $\overline{\mathbf{M}}$ explicitly; see the discussion in [8]. Indeed, the computation and storage of $\overline{\mathbf{M}}$ with $N>75$ is impractical both in terms of computation time and storage requirement on a typical PC. However, we compute $\overline{\mathbf{M}}$ explicitly in this section to evaluate its condition number and to investigate how closely it resembles the identity matrix.

For $N=46$ the matrix $\overline{\mathbf{M}}$ has size $4416 \times 4416$. We shall also consider "truncated" versions of $\overline{\mathbf{M}}$, where only terms with $n \leq N_{\text {tr }}$ are included. A truncated $\overline{\mathbf{M}}$ corresponds to computing the spherical expansion coefficients $A_{n m}$ and $B_{n m}$ of the AUT only up to $n=N_{\text {tr }} \leq N$ based on data sampled at the original rate. In other words, when working with a truncated $\overline{\mathbf{M}}$, we neglect AUT coefficients with $n>N_{\text {tr }}$ while keeping the sampling rate of the higher-order probe output on the scan sphere unchanged. Table 2 shows the size of the truncated normal matrices that will be considered here.

Table 3 shows the condition numbers for the original $\overline{\mathbf{M}}$ matrix and its truncated versions when $\psi=0, \psi=$ $0.5^{\circ}, \psi=1^{\circ}$, and $\psi=2^{\circ}$ probes are employed. Here, the Hertzian-dipole correction factor $\overline{\mathbf{p}}$ is included in the computation of $\overline{\mathbf{M}}$. We see that $\overline{\mathbf{M}}$ is well conditioned in all cases. Moreover, the condition number grows as expected with $\psi$ (the condition number is lowest for the first-order probe). We also see that the condition number decreases with 


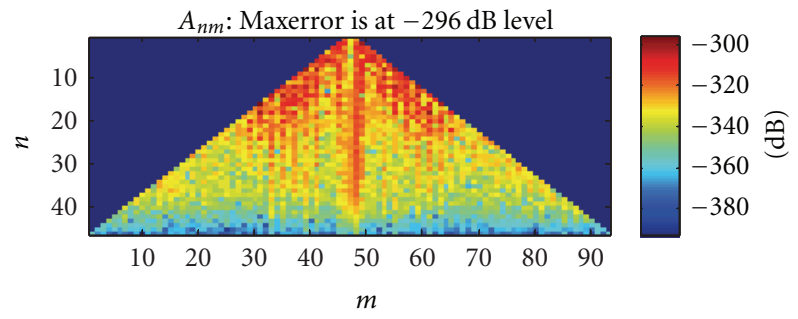

(a)

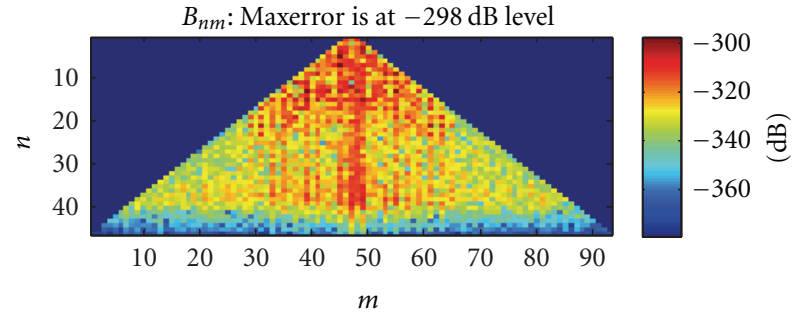

(b)

FIGURE 5: Error plots of the spherical expansion coefficients computed from the higher-order probe output with $\psi=2^{\circ}$ without truncating the $\overline{\mathbf{M}}$ matrix. (a) is $\mathcal{E}_{A_{n m}}^{(46)}$ and (b) is $\mathcal{E}_{B_{n m}}^{(46)}$.

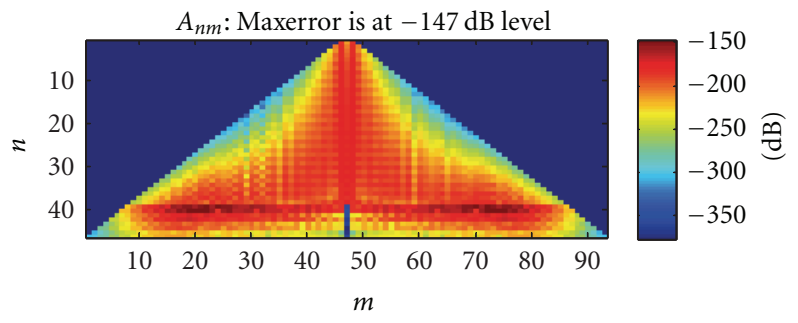

(a)

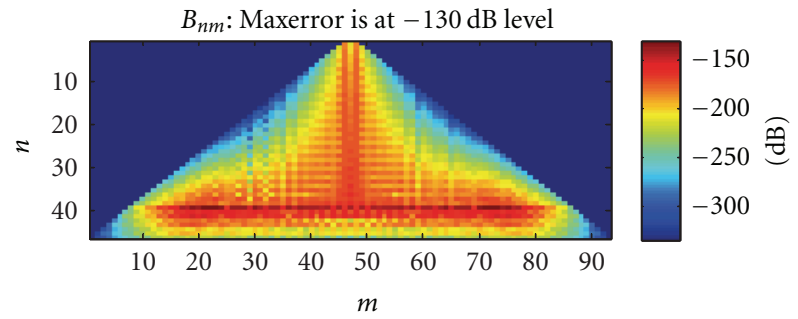

(b)

FIGURE 6: Error plots of the spherical expansion coefficients computed from the higher-order probe output with $\psi=2^{\circ}$ by truncating the $\overline{\mathbf{M}}$ matrix. (a) is $\mathcal{E}_{A_{n m}}^{(38)}$ and (b) plot is $\mathcal{E}_{B_{n m}}^{(38)}$.

TABle 1: Probe-mode power spectrum.

\begin{tabular}{lcccc}
\hline & $\psi=0$ & $\psi=0.5^{\circ}$ & $\psi=1^{\circ}$ & $\psi=2^{\circ}$ \\
\hline Power in modes $|\mu|=1$ & $100 \%$ & $96 \%$ & $84 \%$ & $51 \%$ \\
Power in modes $|\mu| \neq 1$ & $0 \%$ & $4 \%$ & $16 \%$ & $49 \%$ \\
\hline
\end{tabular}

TABLE 2: Size of truncated $\overline{\mathbf{M}}$ matrices.

\begin{tabular}{lc}
\hline$N_{\text {tr }}$ & Size $(\overline{\mathbf{M}})$ \\
\hline 46 & $4416 \times 4416$ \\
44 & $4048 \times 4048$ \\
42 & $3696 \times 3696$ \\
40 & $3360 \times 3360$ \\
38 & $3040 \times 3040$ \\
\hline
\end{tabular}

truncation (the matrix truncated at $n=38$ has a lowest condition number for constant $\psi$ ). This also implies that the $\overline{\mathbf{M}}$ matrix of a given size gets more well conditioned as the sampling rate on the scan sphere is increased. The optimal first-order correction factor discussed in [8] may result in even more well-conditioned normal matrices.

Table 4 shows the corresponding condition numbers obtained by setting $\overline{\mathbf{p}}=\overline{\mathbf{I}}$ in the computation of $\overline{\mathbf{M}}$. In other words, no first-order correction factor is applied. In this case the matrix $\overline{\mathbf{M}}$ can be extremely ill conditioned. For example, when $\psi=2^{\circ}$ and $N_{\mathrm{tr}}=N=46$ the condition number is 1e5. Comparing Tables 3 and 4 clearly demonstrates the benefits of using a proper first-order correction factor represented by the diagonal matrix $\overline{\mathbf{p}}$ containing $U h_{n}^{(1)}(k R)$ and $U g_{n}^{(1)}(k R)$,
Table 3: Condition number of $\overline{\mathbf{M}}$ obtained with Hertzian $\overline{\mathbf{p}}$.

\begin{tabular}{ccccc}
\hline$N_{\text {tr }}$ & $\psi=0$ & $\psi=0.5^{\circ}$ & $\psi=1^{\circ}$ & $\psi=2^{\circ}$ \\
\hline 46 & 14.6 & 26.4 & 31.3 & 56.0 \\
44 & 3.0 & 4.8 & 7.4 & 17.5 \\
42 & 2.2 & 3.4 & 5.3 & 12.8 \\
40 & 1.9 & 2.8 & 4.3 & 10.0 \\
38 & 1.6 & 2.5 & 3.7 & 8.2 \\
\hline
\end{tabular}

Table 4: Condition number of $\overline{\mathbf{M}}$ obtained with $\overline{\mathbf{p}}=\overline{\mathbf{I}}$.

\begin{tabular}{ccccc}
\hline$N_{\text {tr }}$ & $\psi=0$ & $\psi=0.5^{\circ}$ & $\psi=1^{\circ}$ & $\psi=2^{\circ}$ \\
\hline 46 & $3 \mathrm{e} 4$ & $6 \mathrm{e} 4$ & $8 \mathrm{e} 4$ & $1 \mathrm{e} 5$ \\
44 & $1 \mathrm{e} 3$ & $2 \mathrm{e} 3$ & $2 \mathrm{e} 3$ & $4 \mathrm{e} 3$ \\
42 & 195 & 218 & 255 & 412 \\
40 & 46 & 50 & 56 & 85 \\
38 & 19 & 20 & 21 & 30 \\
\hline
\end{tabular}

where $\mathcal{U}$ is given by (14). Also, since $\overline{\mathbf{p}}$ is a diagonal matrix, its associated computational cost is negligible.

Table 5 shows the largest and smallest diagonal values in $\overline{\mathbf{M}}$ (the diagonal elements are positive) for the different probes and different truncation limits under consideration with $\overline{\mathbf{M}}$ computed using a Hertzian $\overline{\mathbf{p}}$. Also shown are the maximum magnitudes of off-diagonal elements for each matrix. The corresponding values for $\overline{\mathbf{p}}=\overline{\mathbf{I}}$ are shown in Table 6. We see that it is the proper Hertzian correction factor which insures that $\overline{\mathbf{M}}$ is close to the identity matrix. In agreement with the condition numbers in Table 3 , the matrix 


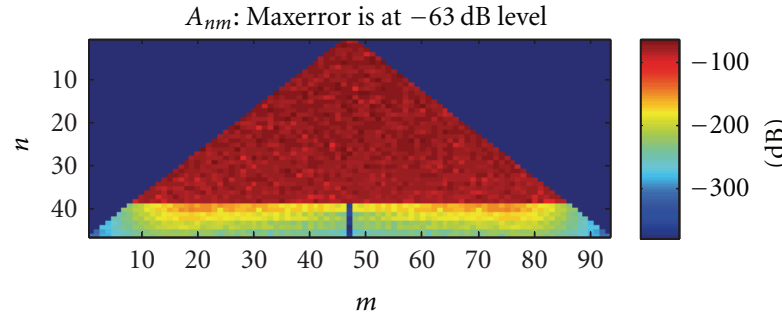

(a)

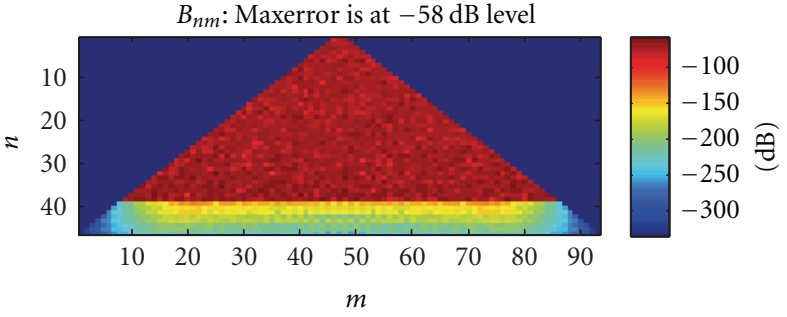

(b)

Figure 7: Error plots of the spherical expansion coefficients computed from the higher-order probe output with $\psi=2^{\circ}$ by truncating the $\overline{\mathbf{M}}$ matrix in the presence of Gaussian probe-output noise at the $-60 \mathrm{~dB}$ level. (a) is $\mathcal{E}_{A_{n m}}^{(38)}$ and (b) plot is $\mathcal{E}_{B_{n m}}^{(38)}$.

TAble 5: Properties of $\overline{\mathbf{M}}$ obtained with Hertzian $\overline{\mathbf{p}}$. D is the maximum value occurring in the diagonal (all diagonal elements are positive), $\mathrm{d}$ is the minimum value occurring in the diagonal, and $\mathrm{O}$ is the maximum magnitude occurring away from the diagonal.

\begin{tabular}{ccccccccccccc}
\hline$N_{\text {tr }}$ & \multicolumn{3}{c}{$\psi=0$} & \multicolumn{3}{c}{$\psi=0.5^{\circ}$} & \multicolumn{3}{c}{$\psi=1^{\circ}$} & \multicolumn{3}{c}{$\psi=2^{\circ}$} \\
& $\mathrm{D}$ & $\mathrm{d}$ & $\mathrm{O}$ & $\mathrm{D}$ & $\mathrm{d}$ & $\mathrm{O}$ & $\mathrm{D}$ & $\mathrm{d}$ & $\mathrm{O}$ & $\mathrm{D}$ & $\mathrm{d}$ & $\mathrm{O}$ \\
\hline 46 & 1.59 & 0.42 & 0.18 & 1.58 & 0.42 & 0.18 & 1.56 & 0.43 & 0.17 & 1.57 & 0.41 & 0.17 \\
44 & 1.10 & 0.90 & 0.074 & 1.09 & 0.90 & 0.071 & 1.07 & 0.91 & 0.066 & 1.09 & 0.91 & 0.092 \\
42 & 1.05 & 0.94 & 0.046 & 1.04 & 0.95 & 0.045 & 1.03 & 0.95 & 0.045 & 1.04 & 0.96 & 0.091 \\
40 & 1.03 & 0.96 & 0.034 & 1.02 & 0.96 & 0.03 & 1.01 & 0.97 & 0.045 & 1.03 & 0.97 & 0.090 \\
38 & 1.02 & 0.97 & 0.027 & 1.02 & 0.97 & 0.026 & 1.01 & 0.97 & 0.044 & 1.02 & 0.97 & 0.089 \\
\hline
\end{tabular}

TABle 6: Properties of $\overline{\mathbf{M}}$ obtained with $\overline{\mathbf{p}}=\overline{\mathbf{I}}$. D is the maximum value occurring in the diagonal (all diagonal elements are positive), $\mathrm{d}$ is the minimum value occurring in the diagonal, and $\mathrm{O}$ is the maximum magnitude occurring away from the diagonal.

\begin{tabular}{ccccccccccccc}
\hline \multirow{2}{*}{$N_{\text {tr }}$} & \multicolumn{3}{c}{$\psi=0$} & \multicolumn{4}{c}{$\psi=0.5^{\circ}$} & \multicolumn{3}{c}{$\psi=1^{\circ}$} & \multicolumn{3}{c}{$\psi=2^{\circ}$} \\
& $\mathrm{D}$ & $\mathrm{d}$ & $\mathrm{O}$ & $\mathrm{D}$ & $\mathrm{d}$ & $\mathrm{O}$ & $\mathrm{D}$ & $\mathrm{d}$ & $\mathrm{O}$ & $\mathrm{D}$ & $\mathrm{d}$ & $\mathrm{O}$ \\
\hline 46 & 4.3 & $2 e-4$ & 0.15 & 4.3 & $2 e-4$ & 0.15 & 4.2 & $2 e-4$ & 0.14 & 4.0 & $2 e-4$ & 0.11 \\
44 & 0.28 & $2 e-4$ & $7 e-3$ & 0.28 & $2 e-4$ & $7 e-3$ & 0.28 & $2 e-4$ & $6 e-3$ & 0.27 & $2 e-4$ & $6 e-3$ \\
42 & $4 e-2$ & $2 e-4$ & $8 e-4$ & $4 e-2$ & $2 e-4$ & $8 e-4$ & $4 e-2$ & $2 e-4$ & $7 e-4$ & $4 e-2$ & $2 e-4$ & $7 e-4$ \\
40 & $9 e-3$ & $2 e-4$ & $2 e-4$ & $9 e-3$ & $2 e-4$ & $2 e-4$ & $9 e-3$ & $2 e-4$ & $2 e-4$ & $9 e-3$ & $2 e-4$ & $2 e-4$ \\
38 & $4 e-3$ & $2 e-4$ & $8 e-5$ & $4 e-3$ & $2 e-4$ & $8 e-5$ & $4 e-3$ & $2 e-4$ & $7 e-5$ & $4 e-3$ & $2 e-4$ & $1 e-4$ \\
\hline
\end{tabular}

$\overline{\mathbf{M}}$ is closest to the identity matrix for the first-order probe. Also, truncating $\overline{\mathbf{M}}$ moves it closer to $\overline{\mathbf{I}}$.

We finally consider a particular AUT consisting of $500 z$ directed Hertzian dipoles randomly distributed in a box with dimensions $-2.8 \lambda<x<2.8 \lambda,-0.03 \lambda<y<0.03 \lambda$, and $-2.8 \lambda<z<2.8 \lambda$, as shown in Figure 4. ( $\overline{\mathbf{M}}$ is of course independent of the AUT; $\overline{\mathbf{M}}$ depends only on the probe, the sampling rate on the scan sphere, and on the highest value of $n$ for which the AUT spherical expansion coefficients are computed.) We computed the exact spherical expansion coefficients $A_{n m}^{\text {exact }}$ and $B_{n m}^{\text {exact }}$ of this AUT from its tangential electric field on the scan sphere using a standard first-order method.

We also computed these AUT expansion coefficients from the output of the $\psi=2^{\circ}$ higher-order probe using the method described in this paper with $N_{\mathrm{tr}}=46$ and $N_{\mathrm{tr}}=38$.
These expansion coefficients are denoted $A_{n m}^{\left(N_{\mathrm{tr}}\right)}$ and $B_{n m}^{\left(N_{\mathrm{tr}}\right)}$. Relative $\mathrm{dB}$ errors are computed as

$$
\begin{aligned}
& \mathcal{E}_{A_{n m}}^{\left(N_{\mathrm{tr}}\right)}=20 \log _{10}\left(\frac{\left|A_{n m}^{\text {exact }}-A_{n m}^{\left(N_{\mathrm{tr}}\right)}\right|}{\max \left(\left|A_{n m}^{\text {exact }}\right|\right)}\right), \\
& \mathcal{E}_{B_{n m}}^{\left(N_{\mathrm{tr}}\right)}=20 \log _{10}\left(\frac{\left|B_{n m}^{\text {exact }}-B_{n m}^{\left(N_{\mathrm{tr}}\right)}\right|}{\max \left(\left|B_{n m}^{\text {exact }}\right|\right)}\right) .
\end{aligned}
$$

Figure 5 shows the relative $\mathrm{dB}$ errors of the AUT spherical expansion coefficients computed without truncating $\overline{\mathbf{M}}$. The error level is at $-300 \mathrm{~dB}$ corresponding to machine precision $(1 e-15)$. Hence, we have numerically validated the higherorder probe-correction scheme. Figure 6 shows the relative $\mathrm{dB}$ errors computed with $N_{\mathrm{tr}}=38$. Here we are neglecting all spherical expansion coefficients with $n>38$. We see that 
the error now is at the $-130 \mathrm{~dB}$ level, which is still very good by anechoic chamber standards (the error level in a good anechoic chamber is $-60 \mathrm{~dB})$.

We next illustrate the benefit of dealing with wellconditioned matrices when the near-field data is noisy. Assume that the truncated $\overline{\mathbf{M}}$ matrix $\left(N_{\mathrm{tr}}=38\right)$ is employed in the presence of Gaussian probe-output noise at the $-60 \mathrm{~dB}$ level. In other words, the exact probe output $\mathrm{W}$ is contaminated by noise as follows:

$$
\mathbf{W}^{(\text {noisy })}=\mathbf{W}+A 10^{-60 / 20}\left[\mathbf{G}_{1}+i \mathbf{G}_{2}\right] / \sqrt{2}
$$

where $\mathbf{G}_{1}$ and $\mathbf{G}_{2}$ are independent Gaussian random vectors with mean and standard deviation equal to zero and one, respectively. The amplitude factor $A$ is the square root of the standard deviation of the exact probe-output power. The noisy data $\mathbf{W}^{(\text {noisy) }}$ is inserted into (12) truncated at $N_{\text {tr }}=$ 38 to get the corresponding set of noisy AUT expansion coefficients. Figure 7 shows that the error level of these AUT expansion coefficients is at $-58 \mathrm{~dB}$. Hence, as a consequence of the well-conditioned $\overline{\mathbf{M}}$ matrix, the error of the computed AUT expansion coefficients is roughly at the same level as the noise present in the probe-output data.

\section{Conclusions}

We evaluated the system-matrix method for higher-order probe correction in spherical scanning using probes with varying higher-order mode pattern energy. The first-order correction factor employed by the method greatly reduces the condition number of the resulting normal matrix, even when half of the probe-pattern energy resides in higher-order modes. Indeed, for the probe with half its energy in the higher-order modes, the first-order correction factor reduced the condition number of a $4416 \times 4416$ normal matrix from $1 e 5$ to 56 .

We also found that even better condition numbers are achieved by truncating the normal matrix; this is equivalent to increasing the sampling rate on the scan sphere for fixed AUT mode truncation number. Increasing the sampling rate by $10 \%$ can significantly reduce the condition number and move the normal matrix closer to the identity matrix. This is important for making the Neumann series solution efficient.

For probes (like the one used in the experimental validation in [8]) with a few percent of the pattern energy in higher-order modes, the first-order correction factor resulted in a $3696 \times 3696$ normal matrix with a condition number of 3.4. The diagonal elements of this normal matrix were in the range from 0.95 to 1.04 , and the maximum magnitude of the off-diagonal elements was 0.045 . Without the firstorder correction factor, the diagonal elements of this normal matrix were in the range from $2 e-2$ to $4 e-2$, and the maximum magnitude of the off-diagonal elements was $8 e-4$. Due to the well-conditioned normal matrices, the method is well behaved in the presence of noise.

\section{Acknowledgment}

The Air Force Office of Scientific Research supported this work.

\section{References}

[1] F. Jensen, Electromagnetic near-field far-field correlations, Ph.D. dissertation, Technical University of Denmark, Lyngby, Denmark, 1970.

[2] P. F. Wacker, "Non-planar near-field measurements: spherical scanning," NBS Internal Report, 1975.

[3] J. E. Hansen, J. Hald, F. Jensen, and F. H. Larsen, Spherical Near-Field Antenna Measurements, Peter Peregrinus, London, UK, 1988.

[4] A. D. Yaghjian and R. C. Wittmann, "The receiving antenna as a linear differential operator: application to spherical near-field measurements," IEEE Transactions on Antennas and Propagation, vol. 33, pp. 1175-1185, 1985.

[5] T. B. Hansen, "Complex-point dipole formulation of probecorrected cylindrical and spherical near-field scanning of electromagnetic fields," IEEE Transactions on Antennas and Propagation, vol. 57, no. 3, pp. 728-741, 2009.

[6] C. H. Schmidt and T. F. Eibert, "Multilevel plane wave based near-field far-field transformation for electrically large antennas in free-space or above material halfspace," IEEE Transactions on Antennas and Propagation, vol. 57, no. 5, pp. 1382-1390, 2009.

[7] T. Laitinen, S. Pivnenko, J. M. Nielsen, and O. Breinbjerg, "Theory and practice of the FFT/matrix inversion technique for probe-corrected spherical near-field antenna measurements with high-order probes," IEEE Transactions on Antennas and Propagation, vol. 58, no. 8, pp. 2623-2631, 2010.

[8] T. B. Hansen, "Spherical near-field scanning with higher-order probes," IEEE Transactions on Antennas and Propagation, vol. 59, no. 11, pp. 4049-4059, 2011.

[9] T. B. Hansen and A. D. Yaghiian, Plane-Wave Theory of TimeDomain Fields, IEEE Press, New York, NY, USA, 1999.

[10] W. C. Chew, J. M. Jin, E. Michielssen, and J. Song, Fast and Efficient Algorithms in Computational Electromagnetics, Artech House, Boston, Mass, USA, 2006.

[11] J. D. Jackson, Classical Electrodynamics, John Wiley and Sons, New York, NY, USA, 2nd edition, 1975. 

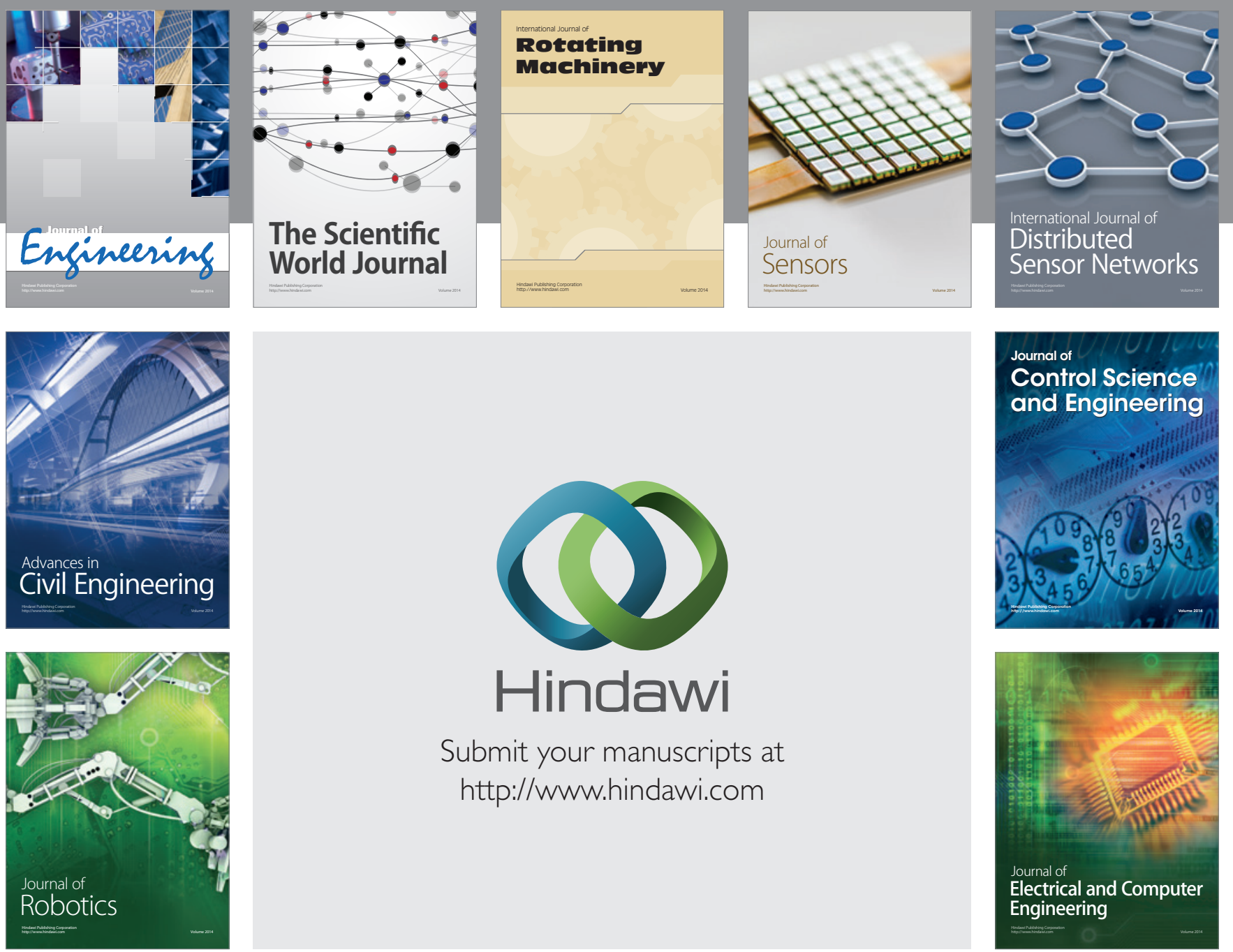

Submit your manuscripts at

http://www.hindawi.com
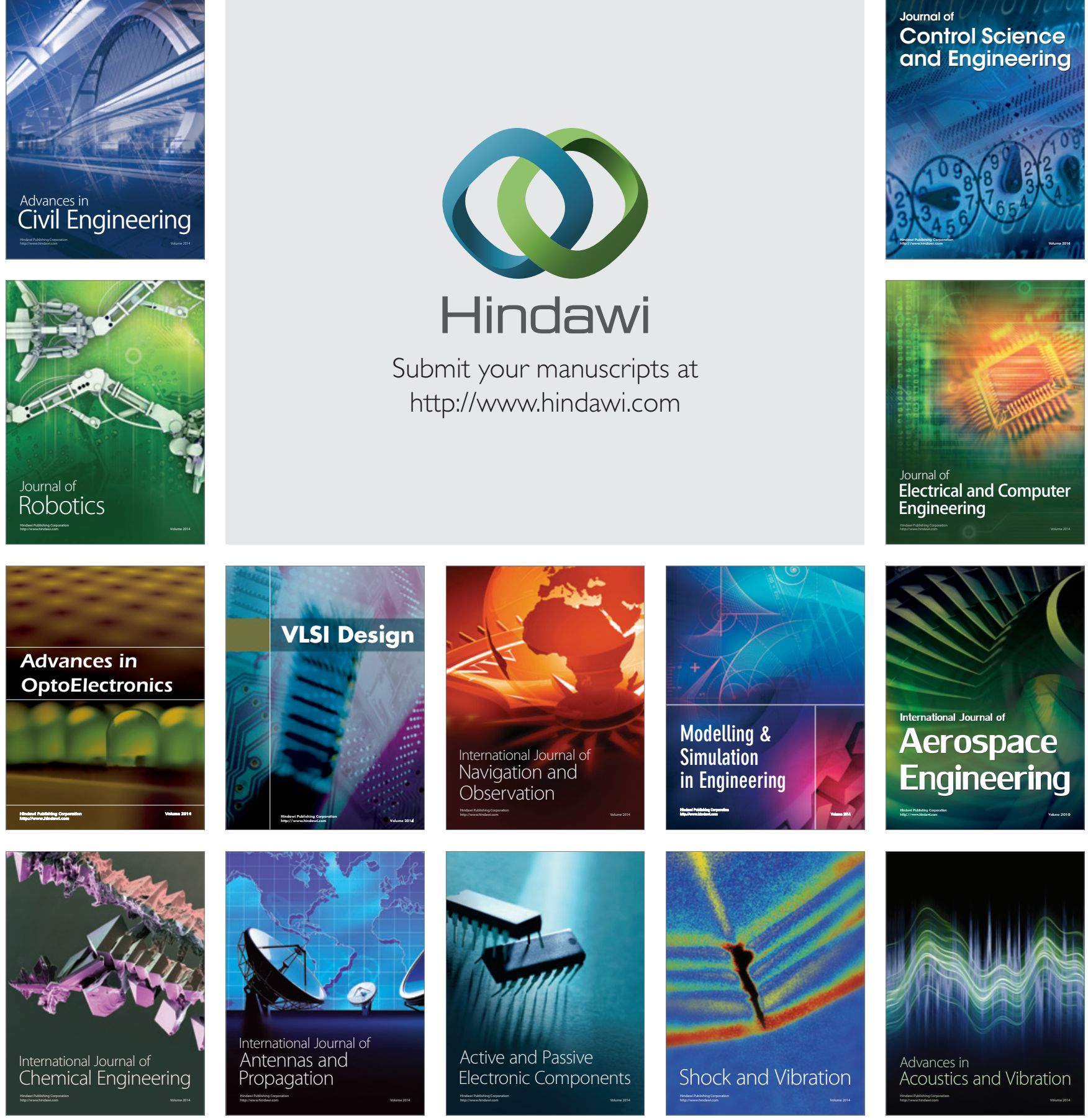